\title{
Graduate student misunderstandings of wave functions in an asymmetric well
}

\author{
C. D. Porter and A. F. Heckler \\ Department of Physics, The Ohio State University, 191 West Woodruff Ave, Columbus, Ohio 43210, USA
}

(Received 20 August 2018; published 11 June 2019)

\begin{abstract}
Quantum mechanics is a notoriously counterintuitive subject within physics and has been the subject of a number of studies at the undergraduate level, and a few pioneering studies at the graduate level. The sketching of wave functions in a confining well is in one sense one of the most basic activities in quantum mechanics. But in another sense, it may be viewed as a rather advanced skill, as it requires the coherent inclusion of a number of details of the wave function, such as wavelength, probability amplitude, and boundary conditions, among others. Although sketching a wave function is not a common activity at the graduate level, a great deal of graduate work is concerned with the aforementioned details, especially boundary conditions. Whether it is seen as a basic skill, or as linked to higher-level understanding, sketching a wave function is an ability that physics graduate students should have. Here, we report on graduate students' ability to sketch wave functions in an asymmetric potential well. We find that the frequency of many errors is not significantly reduced from pretest to posttest, meaning that many errors persist through to the end of graduate quantum mechanics instruction. We find that only 5\% of graduate students tested can sketch the 2nd excited state wave function without errors. We include quantitative and diagrammatic descriptions of student errors covering a broader range of misunderstandings than has previously been identified, and we include interview information that speaks to the persistence of some of the errors.
\end{abstract}

DOI: 10.1103/PhysRevPhysEducRes.15.010139

\section{INTRODUCTION}

Physics education researchers have been studying student difficulties in quantum mechanics for decades (for a review see Ref. [1]), with the vast majority of studies done at the undergraduate level. In this paper we focus on the topic of graduate student understanding and diagrammatic representation of wave functions for an asymmetric potential well. The topic of wave functions in a potential well is fundamental and central in quantum mechanics courses. For example, a common task in upper level undergraduate and graduate courses is that of finding the energy eigenstate wave functions of a particle for a given potential well. Further, a firm understanding of the relationship between potentials and wave functions is important for carrying out calculations and for conceptual understanding in a number of other topics in quantum mechanics, including scattering, one-dimensional, and higher-dimensional bound states, time evolution, the variational method, and the WentzelKramers-Brillouin (WKB) approximation.

Published by the American Physical Society under the terms of the Creative Commons Attribution 4.0 International license. Further distribution of this work must maintain attribution to the author(s) and the published article's title, journal citation, and DOI.
We study student understanding of the diagrammatic representation of the wave function (as opposed to the mathematical expression of the wave function) for a number of reasons. The diagrammatic wave function is a commonly used physical representation in quantum mechanics and appears in a range of popular undergraduate and graduate texts, including those by Liboff, McIntyre, Griffiths, Shankar, and Sakurai [2-6]. Although the end goal in quantum mechanics is often a mathematical calculation, in a number of contexts the physical representation can be an important part of the problem-solving process [7-10]. In the variational method, for example, picking reasonable features like turning points, symmetry, and forbidden regions is critical. The applicability of the WKB approximation depends on how quickly the wavelength $\lambda$ changes on the length scale of $\lambda$ itself [5,11], information which is self-evident in a diagrammatic representation of the wave function. Further, since diagrammatic spatial wave functions provide a description of where particles are likely to be measured, a solid conceptual understanding is likely to be useful to most physics graduate students in their research (both theoretical and experimental). Finally, not only can conceptual understanding and problem solving be aided by an accurate diagram of a wave function, but asking students to produce accurate diagrams of wave functions can be a productive way to assess their understanding. 
There are a few studies that have examined aspects of student understanding of wave functions in and around a potential well [12-18]. In a study of undergraduate physics majors, Goldhaber and Pollock [13] found that a number of misunderstandings become apparent when students draw bound energy eigenstate wave functions. For example, they found that undergraduate students do not clearly understand which conditions require a wave function to equal zero or to exponentially decay to zero. Singh [19] showed that this difficulty still exists among graduate students and showed that a small fraction of students are also confused as to when oscillations in the function occur (drawing oscillations in classically forbidden regions), and how many nodes or antinodes a given wave function should have. There has also been some indication that undergraduate students may sometimes confuse the vertical axes corresponding to potential and probability amplitude (wave function) when they are used on the same plot [20]. These and other points of confusion make it worthwhile to investigate student construction of these important physical representations, and how students' skills with wave functions change over time in a graduate-level course. There have been very few studies of student understanding of quantum mechanics at the graduate level $[19,21,22]$.

Many previous investigations into students' abilities to draw wave functions have used square potential wells (either infinite or finite). This is noteworthy because square wells are introduced even at the freshmen level in some cases [23], and they are ubiquitous in upper-level curricula. Accordingly, most students have seen square well potentials and their energy eigenfunctions on multiple occasions. We hypothesized that some correct responses in the case of a square well may simply be due to recall, and that we may be able to better gauge students' conceptual understanding of energy eigenfunctions by choosing a potential well that they have likely not previously encountered. The asymmetric well has the benefit that it is not often encountered in undergraduate instruction (although it has been used in at least one undergraduate assessment [12]). It is also worth noting that the square wells used in other investigations are piecewise constant. This permits a simple, exact analytical solution, and also permits a student to consider the behavior of the wave function in a constant potential, one region at a time.

The asymmetric well adds an additional layer of complexity: a linearly changing potential with finite walls (see Fig. 1). This added complexity is technically useful for modeling more realistic physical systems that typically have continuously varying potentials. It is also pedagogically useful in that it can be used to assess or facilitate deeper conceptual understanding of important quantitative and qualitative properties of wave functions, such as continuously changing wavelength and amplitude, and the physical reasons for these changes.

With the importance of wave functions in mind, the goals of this inquiry were (i) to replicate and extend the findings

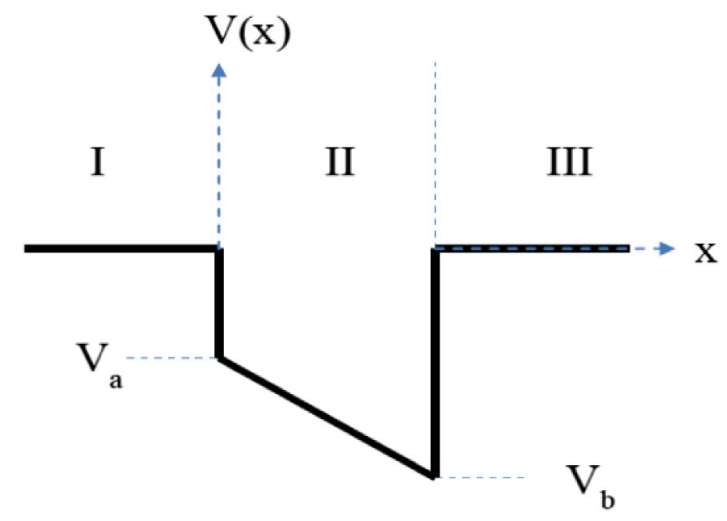

Question

The potential energy diagram for a finite well is shown above. Sketch the wave functions described below. Please clearly label important points and draw wave functions large enough to be easily interpreted!

(a) Draw a qualitative sketch of the ground state in all three regions, assuming $E_{\text {ground }}>V_{\mathrm{a}}$. State any

additional assumptions you are making in doing so. (b) Draw a qualitative sketch of the $2^{\text {nd }}$ excited ( $n=$ 3 , assuming it exists) state in all three regions.

Again, state any assumptions you make.

FIG. 1. Question used in this study. Part (a) was slightly different for the first cohort's pretest, in which the ground state energy was not specified to be above $V_{a}$. The change was made for ease of grading and did not significantly affect student performance.

on student difficulties in understanding the properties of wave functions at the graduate level, and (ii) to determine whether these difficulties or any subset of them are effectively treated by instruction.

\section{METHODS}

This study was carried out with four cohorts of physics graduate students enrolled in the graduate-level quantum mechanics course at The Ohio State University (OSU), between 2014 and 2017. Students were given course credit for participating in a pretest at the beginning of a given semester and a posttest at the end. Credit was based on completeness only. The test consisted of 12-14 conceptual questions on quantum mechanics, covering topics typically addressed in the first semester, such as linear algebra and 1D potential wells. This work focuses on the results from a particular question that was repeated on the pre- and posttests for each cohort, shown in Fig. 1. The sizes of the cohorts varied from 29-41 students. Students were given the chance to opt out of participation in research, but fewer than 5\% did so. Opting out of one test, and significant attrition from the course led to many cases in which preand posttests could not be paired. The total number of pretests, posttests, and paired tests were 131, 102, and 102, 
respectively. Within these, there were instances of students only answering part of the question.

The lectures, homework assignments, and tests were presented in traditional formats for all four cohorts. The only nontraditional aspect of these courses was the addition of voluntary tutorial or guided group work sessions facilitated by one of the authors (C. P.), in consultation with the instructors. Neither asymmetric wells, nor sketches of wave functions were covered in any of the tutorials. So, even though wave functions were discussed, it is not surprising that preliminary analysis showed no statistically significant differences in performance on this question between tutorial participants and nonparticipants. Thus, the tutorials will not be discussed further here. Although there was some variation between cohorts including instructor, book, and grading criteria, these results from a single institution are insufficient to claim the results are representative of physics graduate students in general.

Students completed the assessment working individually in a quiet room. Student progress was timed for the entire assessment, but not for individual questions. Each test took an average of 46 min to complete, and students were cut off after $60 \mathrm{~min}$.

The question given to Cohort 1 on the pretest did not specify that $E_{\text {ground }}>V_{a}$; rather the students were asked to state any assumptions they made. The specification $E_{\text {ground }}>V_{a}$ was added to the question for the posttest given to cohort 1 and all subsequent assessments, in order to make student responses more uniform. There was no statistically significant difference between the groups given the initial and revised phrasings. It will not be discussed further here.

Examples of acceptable correct answers to parts (a) and (b) are shown in Fig. 2. Any answers that satisfied a few key criteria were considered correct. These criteria included continuity of the wave function $\psi(x)$, continuity of the first derivative of the wave function $d \psi(x) / d x=\psi^{\prime}(x)$, exponential decay in the classically forbidden regions, decay to the same line of zero amplitude on each side, correct number of nodes or antinodes, and (in drawings of the 2nd excited state) correct change of amplitude and wavelength with position.

There are subtler issues, such as whether the ground state is peaked slightly to the left or slightly to the right of center

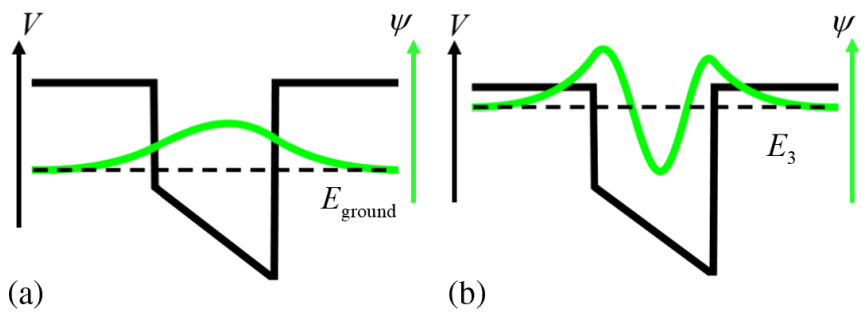

FIG. 2. Examples of acceptable answers to the question parts (a) and (b). in part (a). A full calculation reveals that it is peaked slightly to the right, but this shift is barely perceptible. It is not reasonable to expect students to determine this for the ground state without more time and resources available, nor is it likely that researchers could reliably detect such a minor shift in student sketches. Therefore, answers to part (a) were not judged to be incorrect based on this subtle symmetry issue. In contrast, part (b) should show a significantly higher peak on the left than on the right, and symmetry was considered in the scoring of this part.

We note that part (a) is related to a question used in Ref. [19] (which used a symmetric finite square well). Choosing the finite asymmetric well afforded us some novelty to students, and the opportunity to explore symmetry issues. Additionally, the shape of the asymmetric well allowed us to investigate the extent to which graduate students would use an erroneous line of thinking that we observed anecdotally prior to this study. Specifically, a number of students seemed to think that wave functions must always be peaked where the potential is lowest, an assertion that is reinforced by the ground states of many systems encountered in quantum mechanics coursework. Our experience indicated that the students seemed to associate this idea with the macroscopic (dissipationdriven) phenomenon of particles "settling into an energy minimum." Such thinking might unwittingly facilitate a correct-looking drawing for the ground state of the asymmetric well, but the 2nd excited state would be drawn, according to that specious logic, incorrectly peaked on the right side of the well. We note that careful application of classical physics concepts can still be used to obtain the correct answer for excited states, as must be the case due to the correspondence principle. But for this, one must consider dissipationless oscillation, and one must determine where the classical object would spend the most time, and associate that region with the highest probability [24].

The diagrams produced by students in response to parts (a) and (b) were each scored using a three-level scale, where a " 1 " was entirely correct, a " 0.5 " was mostly correct, and a "0" had multiple errors. Cases of "no response" were tallied separately, not marked as 0 . The frequencies of specific errors were also tracked. The analysis performed here is primarily related to the consistency or improvement in the frequency of errors or misunderstandings. Scoring was carried out by two independent researchers, who assigned the numerical score, and also tallied instances of specific errors. Initial interrater reliability on a test case of 27 students' pretests was low (75\%), prompting improved definitions of grading criteria. Subsequently, interrater reliability on the complete data set was $91 \%$, and was refined through discussion to greater than $98 \%$.

In addition to the collection of written free response data, interviews were also carried out with students in one cohort. All students in that cohort who completed their conceptual 
assessment with time remaining (20 students) were interviewed. The interviews lasted an average of $8 \mathrm{~min}$. Interviews focused on any student errors on the conceptual test that were readily apparent to the researcher conducting the interviews. Although the interviews were not constrained to cover the asymmetric well question, it was the most commonly discussed question. Students were shown their own drawings and were encouraged to talk through their thinking, similar to the think-aloud protocol [25], but with some deviations. For example, there was significant prompting from the interviewer when students displayed the above-described "settling into an energy minimum" misconception, to determine how deeply entrenched it was.

The data collected here are treated primarily in a misconception's framework, as this question was part of a preliminary inquiry to identify what misunderstandings remain after graduate quantum mechanics instruction.

\section{RESULTS AND DISCUSSION}

The two question parts (a) and (b) are referred to here as "ground" and "2nd excited." Table I shows the average scores on the pre- and posttests for each cohort, based on the coarse-grained $0,0.5$, or 1 point scheme. The increase in student score on the ground state from 0.54 to 0.74 was significant $(p<0.001,95 \%$ CI: $0.1-0.7, d=0.47)$. The slight improvement on the 2nd excited state from 0.21 to 0.25 was not quite significant $(p=0.14,95 \%$ CI: $-0.1-0.4, d=0.1)$. The average number of students who drew ground state wave functions in which no error could be reasonably identified was $44 \%$ on the pretests, and $68 \%$ on the posttest, which is a significant increase (McNemar's test, $\chi^{2}=1.5, p=0.001$ ).

Some of these "correct" responses for the ground state may be false positives due to the difficulty in discerning whether a quick sketch was intended to be peaked slightly left, slightly right or perfectly centered. False negatives do not appear since these asymmetry issues were not counted as errors in the grading of the ground state. Almost none of the students correctly drew the second excited state on either the pre (7\%) or posttest (5\%), and these numbers do not reflect a significant change. These data are summarized in Table II.

TABLE I. Average scores using the $0,0.5,1$ grading scheme.

\begin{tabular}{lcccc}
\hline \hline \multicolumn{5}{c}{ Average scores } \\
\hline Cohort & Preground & Postground & $\begin{array}{c}\text { Pre 2nd } \\
\text { excited }\end{array}$ & $\begin{array}{c}\text { Post 2nd } \\
\text { excited }\end{array}$ \\
\hline 1 & 0.43 & 0.44 & 0.34 & 0.16 \\
2 & 0.57 & 0.79 & 0.20 & 0.32 \\
3 & 0.65 & 0.86 & 0.24 & 0.21 \\
4 & 0.50 & 0.78 & 0.13 & 0.26 \\
Total & 0.54 & 0.74 & 0.21 & 0.25 \\
\hline \hline
\end{tabular}

TABLE II. Average percentage of students getting each question part completely correct. Symmetry issues were not counted in the grading of the ground state.

\begin{tabular}{lcccc}
\hline \hline \multicolumn{4}{c}{ Percentage of students answering correctly } \\
\hline Cohort & Preground & Postground & $\begin{array}{c}\text { Pre 2nd } \\
\text { excited }\end{array}$ & $\begin{array}{c}\text { Post 2nd } \\
\text { excited }\end{array}$ \\
\hline Total & 44 & 68 & 7 & 5 \\
\hline \hline
\end{tabular}

The difference in student performance on the ground state and 2nd excited state sketches might indicate that some additional resource is needed to answer the latter. This possibility is discussed further below in the context of specific errors.

\section{Coding of errors:}

Two researchers read through student responses and made separate lists of common incorrect features. The lists were compared and a single master list was developed. After a trial scoring of 27 students' pretests, it became evident that some of the error categories were overlapping or were poorly defined. For example, one researcher initially selected both "oscillations in the forbidden region" and "extra nodes" when a student drew a state with oscillatory behavior in the forbidden region. The other researcher felt that having extra nodes is implicit in having oscillatory behavior outside the well, and did not select extra nodes. Criteria were refined in response to these initial disagreements; they were generally refined to avoid double counting of errors. In the case described above, only oscillations in the forbidden region would be selected, unless there were also extra nodes or antinodes drawn in the classically allowed region.

In all cases, some level of ambiguity was inherent in the hand-drawn sketches. After the initial trial scoring of 27 pretests, it was agreed that an error would only be marked if the researcher could not argue that it might have been accidental. It was with that high bar of error identification that agreement of $91 \%$ was achieved. Using the common errors identified here, future work could be much less ambiguous by having students select from several options in which the errors are unmistakable.

\section{A. Qualitative description of student difficulties}

One common error was to depict the 2nd excited-state wave function with an amplitude peaked to the right in the well. To better understand student thinking behind these diagrams, consider the following two exchanges, each between a graduate student in cohort 2 and an interviewer. Both interviews were conducted in response to the students' drawings of the 2 nd excited-state wave function.

Interview 1

I: Can you explain why you drew this peaked to the right of the center? 
S1: Because... I guess a deeper well corresponds to a higher energy level.

I: The deeper well corresponds to a higher energy?

S1: ... I'm not sure.

I: So, looking at this $n=3$ state that you've drawn. You're saying that when the particle is found over to the right, it had a higher energy prior to measurement than when found on the left?

S1: Um... I'm not sure. Maybe a higher energy relative to the well.

I: Let me ask it slightly differently. In this $n=3$ energy eigenstate that you drew, how does the energy compare..."

S1: Oh! Oh, it's the same! It's an energy eigenstate!

I: Okay. So, can you explain why you drew it peaked to the right of the center?

S1: Um... I'm not sure.

Interview 2

I: So, can you explain why you drew the largest amplitude to the right of the center of the well?

$<$ long pause $>$

S2: Because the probability there is highest.

I: Okay. Why?

S2: Because the energy is lowest there.

I: What energy?

S2: The energy of the particle.

I: I see. So, in this energy eigenstate you've drawn, the energy is lower on the right half of the well?

S2: Yes.

One could interpret that S1 was first trying to speak of kinetic energy rather than total energy, but was unable to properly verbalize this. S1 may also be (incorrectly) associating higher energy with higher amplitude. Even upon resolving some of the confusion upon recognition of the key words "energy eigenstate," S1 still was not able to explain the incorrect diagram drawn, indicating a lack of understanding of the relevant concepts.

S2 was firmer in the belief that energy was lower on the right and may have been associating a classical picture of a particle as being more likely to be found at the lowest portion of the potential well. These interviews are fairly representative of the class as a whole. Some students realized that they were using a classical result, perhaps involving dissipation (i.e., the particle relaxing to the bottom of the well, thus having higher amplitude there) and some realized how to correct their error. Some did not see any discrepancy at all in claiming the energy of the particle is "lower on the right," even upon repetition of the words energy eigenstate.

Both S1 and S2 (and indeed most students interviewed) did clearly indicate that the wave function amplitude is related to the probability of finding a particle in that region. This appeared to be extremely stable in that students never questioned this, even when confronted with other issues. As indicated by the selected interviews, students in general had a more varied mastery of the literal meaning of energy eigenstate. In some cases, students responded to the asymmetric well as though it specified energy, rather than the potential map, whereas other students clearly indicated that the energy of an energy eigenstate is fixed, regardless of where subsequent measurements may localize the particle. Given these observations, it may be productive to consider the amplitude-probability relationship, and energy vs potential distinction as student resources in this context. Further study might allow a better understanding of student errors by using a resources framework.

As shown in Table III, students made a number of errors that one might consider more serious than the subtle issues above, including drawing wave functions with discontinuities in both $\psi(x)$ and in $\psi^{\prime}(x)$. Quite a few students drew wave functions that continued to oscillate in the classically forbidden walls of the well, despite clearly drawing a confined bound state inside the well. A number of students seemed to draw highly oscillatory wave functions with six nodes or more, despite the fact that both " 2 nd excited state" and " $(n=3)$ " appear in the question stem.

The asymmetric nature of the well led to one completely unanticipated student error, which we refer to as "follow slant" in Table III. In this error, students drew a wave function resembling the eigenfunctions of an infinite square well but anchored the endpoints of this function on the lower corners of the well. In this way the wave function follows the inclined bottom of the potential well. Students did this for both ground and excited states. This error appears to stem from students confusing the two sets of axes implicitly present in many such potential well or wave function diagrams. The slanted bottom of the well is a line indicating potential energy as a function of position. The wave function drawn superposed over the well is a diagram of probability amplitude as a function of position. The two vertical axes do not necessarily have any direct relationship. It appears that students might be interpreting a lowest point in the plot of energy as also being the "zero" of probability amplitude. This assertion is supported by student interviews like the following.

Interview 3:

I: Can you tell me why you drew this state the way you did?

S: That's just how I learned it.

I: Sure. But can you explain, for example, why the end points are where they are?

S: Because the wave function has to go to zero at the edges.

I: And zero is at the bottom of the well?

S: Yes. Well, by convention. I can always shift the potential by any constant.

I: Sure. Sure. But the common convention is to put zero here and here? (Note: gesturing to the bottom of the well, on each side, at different potentials)

S: Yeah.

Relatively few students made the "follow slant" error (around 10\%). But the occurrences of this error spanned 
TABLE III. Common errors in drawing the wave function in the asymmetric well. Some could only be marked as errors in drawings of the 2nd excited state. Graphical representations are tracing reproductions by investigators.

\begin{tabular}{|c|c|c|c|c|c|}
\hline Abbrev. & Description & Example & Abbrev. & Description & Example \\
\hline Prob. & $\begin{array}{l}\text { The wave function is } \\
\text { drawn like a } \\
\text { probability }\left(|\psi|^{2}\right)\end{array}$ & & Extra nodes & $\begin{array}{l}\text { There are too many } \\
\text { nodes for the wave } \\
\text { function described }\end{array}$ & \\
\hline Sym. & $\begin{array}{l}\text { The wave function is } \\
\text { symmetric in the } \\
\text { classically allowed } \\
\text { region }\end{array}$ & & Asym. decay & $\begin{array}{l}\text { In the classically } \\
\text { forbidden region, } \\
\text { the decay is to } \\
\text { different zeros }\end{array}$ & \\
\hline Wrong sym. & $\begin{array}{l}\text { The maximum in the } \\
\text { probability } \\
\text { amplitude appears } \\
\text { to the right of the } \\
\text { center (part b) }\end{array}$ & & Follow slant & $\begin{array}{l}\text { The wave function } \\
\text { appears to follow } \\
\text { the slant of the } \\
\text { potential well }\end{array}$ & \\
\hline Disc. $\psi$ & $\begin{array}{l}\text { A discontinuity } \\
\text { appears in } \psi(\mathrm{x})\end{array}$ & & Osc. forb. & $\begin{array}{l}\psi(\mathrm{x}) \text { is oscillatory in } \\
\text { the classically } \\
\text { forbidden region }\end{array}$ & \\
\hline Disc. $\psi^{\prime}$ & $\begin{array}{l}\text { A discontinuity } \\
\text { appears in } \psi^{\prime}(x)\end{array}$ & & Too few nodes & $\begin{array}{l}\text { There are too few } \\
\text { nodes for the wave } \\
\text { function described }\end{array}$ & \\
\hline $\begin{array}{l}\psi=0 \text { at } \\
\text { bound. }\end{array}$ & $\begin{array}{l}\text { Wave function goes } \\
\text { immediately to zero } \\
\text { at the well's edge }\end{array}$ & & Amp. $\lambda$ err. & $\begin{array}{l}\text { The amplitude is } \\
\text { inconsistent with } \\
\text { the wavelength }\end{array}$ & \\
\hline
\end{tabular}

multiple cohorts and the error was robust among the few students who were interviewed about this. Such confusion of the two implied axes was noted as early as 2003 [20] for undergraduates.

The interpretation of the student error as being related to axis confusion is further supported by student diagrams in which the asymptotic zero-amplitude line was labeled " $V=0 "$ Also, closely related to this error was one in which students drew wave functions decaying to different zero-amplitude lines on the right and left of the well (see Table III).

There were a number of other subtler errors such as drawing 2nd excited state wave functions in which the changes in amplitude and wavelength were inconsistent. When the amplitude of a wave function increases (as a function of position), the wavelength should also increase. Many students drew amplitudes that changed across the well, but drew the wavelengths as constant, or drew both as being constant. Others switched the relationship between wavelength and amplitude, drawing very short wavelengths where the amplitude was very large. This class of errors warrants some additional breakdown in that some students seemed to be aware that either the wavelength or the amplitude must vary with position, and were simply inconsistent in their application of this knowledge, whereas others drew the wavelength and amplitude as constant across the well. Such errors are clearly more relevant for the 2nd excited state, and we restrict the discussion here to that part. Those drawings in which the variation in wavelength and in amplitude did not match each other, or did not match the well accounted for 22 of the 51 "Amp. $\lambda$ err." errors noted on the pretest, and 36 of 59 such errors on the posttest. The remainder of the errors came from treating the amplitude and wavelength as constant. Although the shift seems to suggest an increased awareness of wavelength or amplitude dependence on potential from pretest to posttest, the change was not statistically significant for either subset of errors, according to McNemar's test.

In one of the most common errors noted on the drawings of the 2nd excited state wave function, students drew wave function troughs at the same vertical level as the exponential decays off to the left or right. In other words, it was not the nodes in $\psi(x)$ that were drawn at $\psi(x)=0$, but rather the minima of $\psi(x)$. As a result, the wave function sketches appeared more like probability sketches [involving $|\psi(x)|^{2}$ ] of the 1st excited state. Although there could certainly be student confusion about the relationship between $\psi(x)$ and probability, there is no indication that students were 
confused about which was being asked for by the question stem. In particular, one student drew and labeled both $\psi(x)$ and $|\psi(x)|^{2}$, even though the latter had not been requested. The student drew both curves never crossing the zeroamplitude line, as described above.

\section{B. Quantitative description of student difficulties}

Figure 3 presents the percentages of occurrence of the most common errors made for the ground and excited states. The errors have been roughly sorted into groups convenient for discussion. Those errors furthest to the left may be reduced during the course. The errors toward the center of Fig. 3 (between the bold dashed lines) appear less well addressed by instruction. The errors to the right are those that one would expect to apply differently to parts (a) and (b) of the question. For example, it is easy to imagine a student drawing an improper relationship between the amplitude and wavelength in the 2 nd excited state. It is difficult to imagine that mistake being evident in a drawing of the ground state, as it would require at least one additional error (drawing too many nodes or antinodes for the ground state). We will refer to those errors that are plausibly equally applicable to both the ground and 2nd excited states as "type I" errors, and those that apply differently to the two states as "type II" errors.

It must be emphasized that Fig. 3 is made up of all pretest data and all posttest data, not merely within-student paired pretests and posttests. This has been done to emphasize the presence of various errors (some of which are slightly suppressed by the removal of unpaired data points). In preand postanalysis described below (McNemar tests), only paired pretests and posttests were used. Paired and unpaired data were qualitatively similar, with differences mostly observable in very low- $N$ errors, such as discontinuities in the wave function or its first derivative.

There were variations in the frequency of some errors between cohorts. However, a repeated measures analysis in SPSS using cohort as a between-subjects factor showed no statistically significant differences in error frequency. Hence all cohorts are grouped together in our analysis.

An important question is whether or not student responses, and specific student mistakes on this assessment are indicative of robust misunderstandings or are simply the results of imprecise, neglectful, or inadvertent answering.

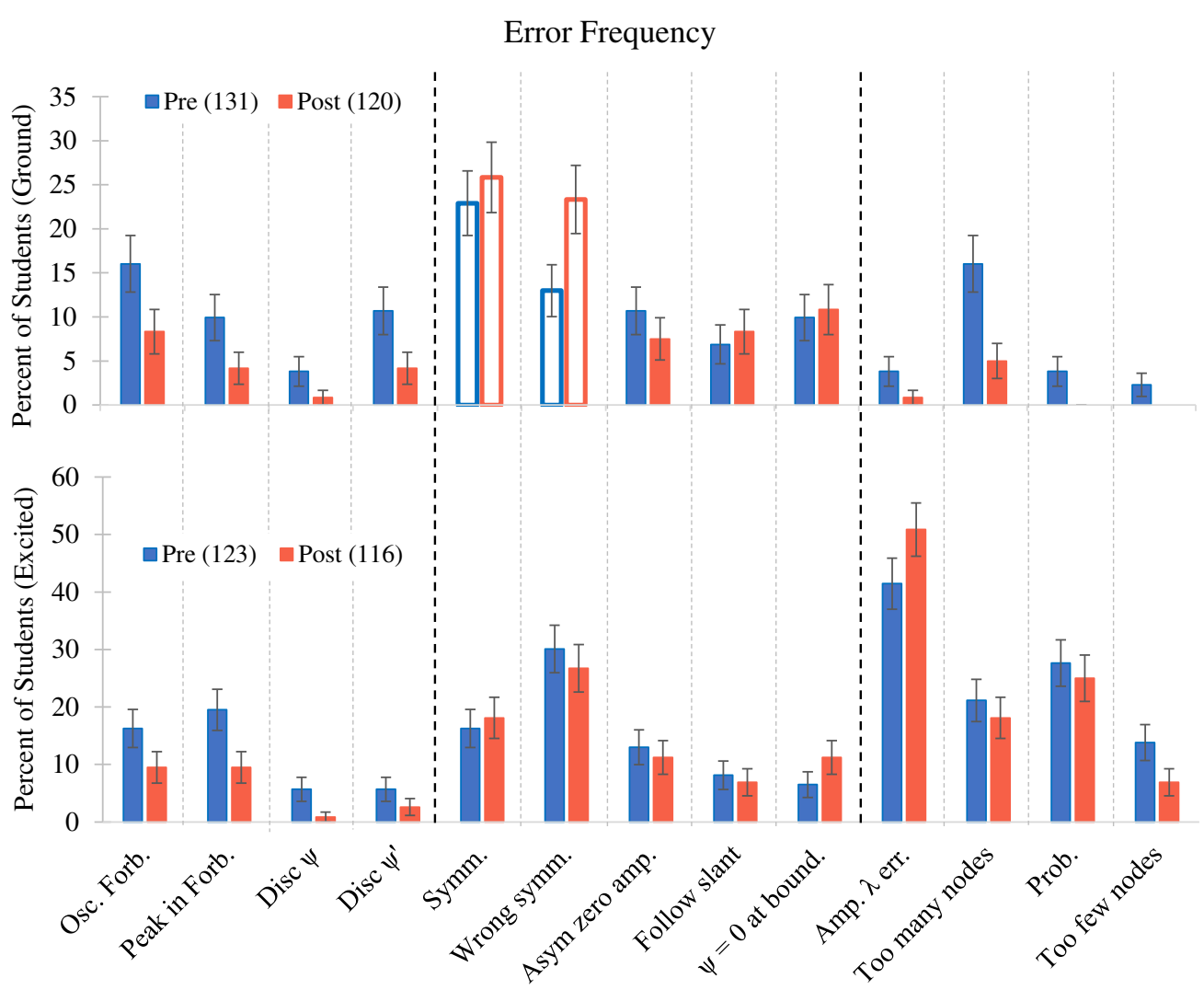

FIG. 3. Pre- and postcomparison of student errors on the drawing of ground state wave functions and 2nd excited state wave functions. In general, errors are not mutually exclusive. The bars for the categories "Sym." and "Wrong sym." are not shaded in for the ground state, indicating that they were not considered in the grading of student responses for the ground state. Error bars represent binomial error, treating each student's answer as a separate Bernoulli trial, and treating pretest groups and posttest groups entirely separately. The data correspond to all pretest data and all posttest data, not restructured to within-student paired pretests and posttests. 
Our interviews with students, including examples from the previous section, often indicated that the errors were not inadvertent errors or simple oversights. More formally, correlation coefficients were calculated to determine whether a student who made an error in the ground state was likely to make the same mistake on the 2 nd excited state. In this case the relevant correlation coefficient is the $\phi$ (phi) coefficient, since the within-student data being examined are binary (they either made an error or did not). The coefficients are shown in Table IV, as are 95\% confidence intervals.

As discussed above, there are a few categories in which one would not expect the errors to be correlated. These are in a separate section of Table IV. The upper portion shows type I errors: those that could reasonably be made and identified in both the ground state and 2nd excited state, and which students may consistently apply in both contexts. In the lower portion are type II errors: those that cannot be made or identified in the ground state without additional errors. For example, it is not possible for the ground state wave function to show inconsistent changes in wavelength and amplitude, unless a student also draws too many nodes or antinodes. In all cases type I error correlations were found to be significant; $p$ values calculated using a Fisher's exact test in SPSS were at or below 0.001, and remain significant after a post hoc Bonferroni correction. The correlations for type I errors were generally moderate to large $(\varphi>0.7$ is often used as a benchmark for a very strong correlation, with 0.3-0.7 being moderate to substantial). The correlations for type II errors were generally smaller, and not statistically significant, as expected.

TABLE IV. Correlations ( $\varphi$ coefficients) between common student errors on the ground state and 2 nd excited state. Type I errors are those that are equally applicable to the ground and 2nd excited states, while type II errors are those that would be impossible to make or identify in the ground state, unless additional errors were made.

\begin{tabular}{lcccc}
\hline \hline & \multicolumn{2}{c}{$\begin{array}{c}\text { Ground or Exc. } \\
\text { Corr. (Pretest) }\end{array}$} & \multicolumn{2}{c}{$\begin{array}{c}\text { Ground or Exc. } \\
\text { Corr. (Posttest) }\end{array}$} \\
\hline Type I errors & $\phi$ & $95 \%$ CI & $\phi$ & $95 \%$ CI \\
Oscill. in forb. & 0.75 & $0.65-0.81$ & 0.57 & $0.42-0.67$ \\
Peak in forb. & 0.30 & $0.12-0.45$ & 0.35 & $0.18-0.5$ \\
$\Psi=0$ at bound. & 0.69 & $0.58-0.77$ & 0.87 & $0.81-0.9$ \\
Disc. $\Psi$ & 0.84 & $0.77-0.88$ & $*$ & $*$ \\
Disc. $\Psi^{\prime}$ & 0.35 & $0.18-0.5$ & 0.50 & $0.35-0.62$ \\
Extra nodes & 0.57 & $0.42-0.67$ & 0.23 & $0.05-0.39$ \\
Asym. decay & 0.57 & $0.49-0.72$ & 0.51 & $0.36-0.63$ \\
Follow slant & 0.89 & $0.83-0.91$ & 0.77 & $0.67-0.83$ \\
Type II errors & $\varphi$ & $95 \%$ CI & $\varphi$ & $95 \%$ CI \\
Symmetric & 0.26 & $0.08-0.42$ & 0.29 & $0.1-0.44$ \\
Wrong symm. & -0.16 & $-0.34-0.02$ & -0.28 & $-0.43-0.1$ \\
Amp. $\lambda$ wrong & 0.03 & $-0.15-0.2$ & 0.09 & $-0.09-0.26$ \\
Prob. & 0.14 & $-0.06-0.4$ & $*$ & $*$ \\
\hline \hline
\end{tabular}

This consistent pattern of answering lends support to the idea that the incorrect student answering was due to either the consistent application of an incorrect understanding or some persistent automatic processes cued by the asymmetric well context.

Inspection of Fig. 3 indicates that some errors may be reduced during the graduate quantum mechanics course, such as for discontinuities in $\psi^{\prime}(x)$. A great many errors do not appear to have been significantly reduced in Fig. 3, such as drawing the wave functions going to zero at the boundaries. However, Fig. 3 includes students who only took the pretest, only took the posttest, or took both. The question of whether errors were significantly affected by instruction must be answered using the subset of students for which there exist paired pretests and posttests. Pulling from all four cohorts, 102 such pre- and postpairs exist. Individual McNemar's tests of marginal homogeneity indicate that these errors are not statistically reduced by instruction (neither before nor after a post hoc correction).

\section{CONCLUSIONS}

Our findings indicate that many misunderstandings about wave functions persist throughout students' graduate experience, even after instruction. By the end of their firstyear experience, only about $5 \%$ of students could correctly draw the 2 nd excited state in the asymmetric well. Figure 3 shows which of the errors contributed to this poor performance. Only one error seemed to become more prevalent after instruction: drawing an incorrect relationship between the amplitude and wavelength. All others appear in Fig. 3 to be reduced or remain steady. However, restricting the analysis to students who participated in both the pretest and posttest reveals that the reductions are not statistically significant. The decrease is made up of many students correcting the error by the time the posttest comes, and slightly fewer students making the error for the first time on the posttest.

As mentioned, there are relatively few opportunities for comparison with existing research. But the study by Singh [19] did give numerical values for instances of three errors noted in this study, but only prior to instruction: oscillation in the forbidden region, extra nodes, and $\psi=0$ at the boundary. Although differences in the wells could explain significant numerical disagreement in these error frequencies, the numbers from Ref. [19] are qualitatively consistent with our findings. This is apparent in Table V. Numbers both from our study and Ref. [19] are for the ground state.

In the similar prior study [19], no posttest data were taken. From the posttest data in this work, it is clear that these errors are treated to varying extents, but any reduction is not statistically significant. This is the first indicator that the missing basic skills with wave functions may not be adequately addressed during graduate physics instruction.

The asymmetric well was instrumental in discovering many errors not previously reported at the graduate level. 
TABLE V. Percentages of students making errors on the ground states in the pretests given by Singh [19] and in this work. Uncertainties were not available from Ref. [19].

\begin{tabular}{lcc}
\hline \hline Error & Singh [19] & Present work \\
\hline Osc. forb. & $8 \%$ & $16 \pm 4 \%$ \\
Extra nodes & $20 \%$ & $16 \pm 3 \%$ \\
$\psi=0$ at bound. & $8 \%$ & $10 \pm 3 \%$ \\
\hline \hline
\end{tabular}

Using a symmetric well, it would not have been possible to identify the errors labeled "follow slant" and "Asym. Decay."

One point that may be very relevant to instruction is that students made a wide variety of errors, and that most students made multiple errors on any given part of the question. For example, on the posttest, $40 \%$ of students made multiple errors on the drawing of the 2 nd excited state. The fact that students made errors consistently means that students either have stable misunderstandings about wave functions, or are systematically failing to include what they know about wave functions in sketches. Either of these could adversely affect their use of wave function sketches in solving, for example, problems involving the variational method. What this broad range of errors means for instruction is an open question.

The results reported here came from four different cohorts, using a variety of source material, being taught by three different faculty members, and having varying pass or fail rates. The student body was diverse, including many international students, and many students from underrepresented minorities in physics. This variety raises the possibility that these results may be indicative of student misunderstandings in similar large public universities throughout the U.S. Collaborations are currently underway with three other midwestern universities to address this possibility.

As mentioned previously, a number of approximation methods and higher-level skills in graduate physics build on wave functions as representations of quantum states. An open question is whether proficiency at the higher skills actually correlate to a students' ability to accurately depict a wave function. For example, as mentioned in the introduction, an understanding of wave functions can play an important role in the WKB approximation, where the relationship between $\lambda$ and the rate of change of $\lambda$ is used as a criterion for the approximation's applicability. Students who drew the 2nd excited state as having a constant wavelength may have considerable difficulty applying this criterion. Also, the boundary matching process that one typically employs at classical turning points in the WKB approximation is necessary because the wavelength diverges in the classically forbidden region. This process might be extremely confusing to students who drew oscillatory behavior in the classically forbidden region. But these difficulties with the WKB method and other approximation methods do not necessarily follow from our findings. In future work, we hope to check for correlations between basic knowledge and skills involving wave functions and higher-level skills such as approximation methods.

\section{ACKNOWLEDGMENTS}

The authors wish to thank The Ohio State University physics graduate students for participation in this study. We thank The Ohio State University Physics faculty for their useful discussions, suggestions, and endorsement of our study. We wish to acknowledge the contribution of Dr. Abigail Bogdan, who helped review and analyze the first year of data, and helped with early drafts of the assessment, as well as Srividya Suresh, who assisted in grading. We also wish to thank The Ohio State University Physics M.S. to Ph.D. Bridge Program and the American Physical Society Bridge Program, for supporting the role of our Physics Education Research group in advanced physics and graduate physics courses at The Ohio State University.

Primary funding support for this research was provided by the National Science Foundation NRT-IGE program under Grant No. 1735027. We also acknowledge partial support from the Center for Emergent Materials: an NSF MRSEC under Grant No. DMR-1420451. Any opinions, findings, and conclusions or recommendations expressed in this material are those of the author(s) and do not necessarily reflect the views of the National Science Foundation.
[1] C. Singh and E. Marshman, Review of student difficulties in upper-level quantum mechanics, Phys. Rev. ST Phys. Educ. Res. 11, 020117 (2015).

[2] R. Liboff, Introductory Quantum Mechanics (AddisonWesley, Boston, MA, 2003).

[3] D. H. McIntyre, Quantum Mechanics (Pearson AddisonWesley, San Francisco, CA 2012).
[4] D. J. Griffiths, Introduction to Quantum Mechanics (Prentice Hall, Upper Saddle River, NJ 1995), preface.

[5] R. Shankar, Principles of Quantum Mechanics 2nd ed. (Springer, New York, NY 1994).

[6] J. Sakurai and Jim Napolitano, Modern Quantum Mechanics, revised 2nd ed. (Cambridge University Press, Cambridge, England, 2018). 
[7] J. H. Larkin, The role of problem representation in physics, Mental Models (Psychology Press, New York 2014), pp. 83-106.

[8] P. B. Kohl and N. D. Finkelstein, Effects of representation on students solving physics problems: A fine-grained characterization, Phys. Rev. ST Phys. Educ. Res. 2, 010106 (2006).

[9] P. B. Kohl and N. D. Finkelstein, Student representational competence and self-assessment when solving physics problems, Phys. Rev. ST Phys. Educ. Res. 1, 010104 (2005).

[10] D. Rosengrant, E. Etkina, and A. Van Heuvelen, An overview of recent research on multiple representations, AIP Conf. Proc. 883, 149 (2007).

[11] For a review, see R. B. Dingle, Asymptotic Expansions: Their Derivation and Interpretation (Academic Press, Cambridge, MA 1973).

[12] H. R. Sadaghiani and S. J. Pollock, Quantum mechanics concept assessment: Development and validation study, Phys. Rev. ST Phys. Educ. Res. 11, 010110 (2015).

[13] S. Goldhaber, S. Pollock, M. Dubson, P. Beale, and K. Perkins, Transforming upper-division quantum mechanics: Learning goals and assessment, AIP Conf. Proc. 1179, 145 (2009).

[14] S. Wuttiprom, M. D. Sharma, I. D. Johnston, R. Chitaree, and C. Soankwan, Development and use of a conceptual survey in introductory quantum physics, Int. J. Sci. Educ. 31, 631 (2009).

[15] P. Jolly, D. Zollman, S. Rebello, and A. Dimitrova, Visualizing motion in potential wells, Am. J. Phys. 66, 57 (1998).

[16] S. B. McKagan, K. K. Perkins, M. Dubson, C. Malley, S. Reid, R. LeMaster, and C. E. Wieman, Developing and researching PhET simulations for teaching quantum mechanics, Am. J. Phys. 76, 406 (2008).

[17] C. Baily and N.D. Finkelstein, Interpretive themes in quantum physics: Curriculum development and outcomes, AIP Conf. Proc. 1413, 107 (2012).

[18] C. Singh, Student understanding of quantum mechanics, Am. J. Phys. 69, 885 (2001).

[19] C. Singh, Student understanding of quantum mechanics at the beginning of graduate instruction, Am. J. Phys. 76, 277 (2008).

[20] J. T. Morgan, M. C. Wittmann, and J. R. Thompson, Student understanding of tunneling in quantum mechanics: Examining interview and survey results for clues to student reasoning, AIP Conf. Proc. 720, 97 (2004).

[21] L. D. Carr and S. B. McKagan, Graduate quantum mechanics reform, Am. J. Phys. 77, 308 (2009).

[22] C. D. Porter, A. Bogdan, and A. Heckler, Student understanding of potential, wavefunctions, and the Jacobian in hydrogen in graduate-level quantum mechanics, Proceedings of the 2016 Physics Education Research Conference, Sacramento CA (AIP, New York, 2016), pp. 244-247, https://www.per-central.org/items/detail .cfm?ID=14239\&Relations $=1$.

[23] D. Halliday, R. Resnick, and J. Walker, Fundamentals of Physics Extended, 9th ed. (Wiley, Hoboken, NJ, 2011), Chap. 38.

[24] L. Bao, P. Jolly, and E. F. Redish, Student difficulties with quantum mechanics, AAPT Announcer 26, 70 (1996).

[25] M. T.H. Chi, The Thinking Aloud Method (Academic Press, London 1994), Chap. 1. 